\title{
JACOBIANS AMONG ABELIAN THREEFOLDS: A FORMULA OF KLEIN AND A QUESTION OF SERRE
}

\author{
Gilles Lachaud, Christophe Ritzenthaler, And Alexey Zykin
}

\begin{abstract}
Let $(A, a)$ be an indecomposable principally polarized abelian threefold defined over a field $k \subset \mathbb{C}$. Using a certain geometric Siegel modular form $\chi_{18}$ on the corresponding moduli space, we prove that $(A, a)$ is a Jacobian over $k$ if and only if $\chi_{18}(A, a)$ is a square over $k$. This answers a question of J.-P. Serre. Then, via a natural isomorphism between invariants of ternary quartics and Teichmüller modular forms of genus 3 , we obtain a simple proof of Klein formula, which asserts that $\chi_{18}(\operatorname{Jac} C, j)$ is equal to the square of the discriminant of $C$.
\end{abstract}

\section{Introduction}

Let $\mathrm{A}_{3}$ be the moduli stack of principally polarized abelian schemes $(A, a)$ of relative dimension 3 and $M_{3}$ be the moduli stack of smooth and proper curves of genus 3 . The first aim of this article is to answer the following question of Serre [20]: If $k$ is a subfield of $\mathbb{C}$, and if $(A, a) \in \mathrm{A}_{3} \otimes k$, under what conditions is it isomorphic over $k$ to a polarized Jacobian? If $k=\bar{k}$, this is the case if and only if $(A, a)$ is indecomposable, according to Hoyt [9] and Oort and Ueno [18]. We henceforth assume $(A, a)$ indecomposable, and isomorphic over $\bar{k}$ to the principally polarized Jacobian ( Jac $C, j$ ) of a curve $C / \bar{k}$ of genus 3 . For a general field $k \subset \mathbb{C}$, the answer is given by a particular Siegel modular form $\chi_{18}$ of genus 3 . This form is actually defined up to a multiplicative constant by the product of the 36 Thetanullwerte with even characteristics. Our main result (Th. 1.3.3) is the following criterion: $(A, a)$ is isomorphic over $k$ to $(\operatorname{Jac} C, j)$ if and only if $\chi_{18}(A, a)$ is a square over $k$. This was suggested by Serre in [20] and proved in [15] by the first two authors for a 3-dimensional family of abelian varieties. This square appears due to the following fact: by taking the inverse image under the Torelli morphism $t: C \mapsto(\operatorname{Jac} C, j)$ (with $j$ the canonical polarization), we get an element $t^{*} \chi_{18}$ in the algebra of Teichmüller modular forms over $k$, which turns out to be a square, according to Ichikawa [10]. The equivalence is then obtained by the action of quadratic twists on geometric Siegel modular forms.

The second part of the article uses a natural isomorphism between the algebra of invariants on the space of ternary quartic forms with non zero discriminant and the algebra of Teichmüller modular forms on the space of non hyperelliptic curves of genus 3 . Hence, the form $t^{*} \chi_{18}$, restricted to nonsingular non hyperelliptic curves, can be interpreted as an invariant and this provides a simple proof of a formula of Klein, which asserts that $\chi_{18}(\mathrm{Jac} C, j)$ is the square of the discriminant of $C$ (Th. 2.2.3). The original relevance of Klein's formula for the above criterion was one of Serre's

Received by the editors August 7, 2009. 
insights.

This article is organized in two sections. In $\S 1.1$, we review the necessary elements from the theory of Siegel and Teichmüller modular forms, then in $\S 1.2$ we introduce the action of isomorphisms and see how the action of twists is reflected on the values of modular forms, and we prove our main result in $\S 1.3$. The second section deals with invariants: in $\S 2.1$, we give a geometric description of invariants of ternary forms, and in $\S 2.2$, we prove Klein's formula. Finally, in $\S 2.3$ we discuss the reasons behind the failure of a straightforward generalization of the theory in higher dimensions.

We would like to thank J.-P. Serre and S. Meagher for fruitful discussions, and Y. F. Bilu and X. Xarles for their help in $\S 2.3$.

\section{Modular forms and abelian threefolds}

1.1. Siegel and Teichmüller modular forms. References for the following results are [3], [4], [5], [7]. Let $g \geq 2$ be an integer and $\mathrm{A}_{g}$ be the moduli stack of principally polarized abelian schemes of relative dimension $g$. Let $\pi: \mathrm{V}_{g} \longrightarrow \mathrm{A}_{g}$ be the universal abelian scheme and $\pi_{*} \Omega_{\mathrm{V}_{g} / \mathrm{A}_{g}}^{1} \longrightarrow \mathrm{A}_{g}$ the rank $g$ bundle, usually called Hodge bundle, induced by the relative regular differential forms of degree one on $\mathrm{V}_{g}$ over $\mathrm{A}_{g}$. The relative canonical bundle over $A_{g}$ is the line bundle

$$
\boldsymbol{\omega}=\bigwedge^{g} \pi_{*} \Omega_{\mathrm{V}_{g} / \mathrm{A}_{g}}^{1} .
$$

Let $R$ be a commutative ring and $h$ be a positive integer. A geometric Siegel modular form of genus $g$ and weight $h$ over $R$ is an element of the $R$-module

$$
\mathbf{S}_{g, h}(R)=\Gamma\left(\mathrm{A}_{g} \otimes R, \boldsymbol{\omega}^{\otimes h}\right) .
$$

One proceeds similarly with curves. Let $\mathrm{M}_{g}$ denote the moduli stack of smooth and proper curves of genus $g$. Let $\pi: \mathrm{C}_{g} \longrightarrow \mathrm{M}_{g}$ be the universal curve, and let $\boldsymbol{\lambda}$ be the invertible sheaf associated to the Hodge bundle on $\mathrm{M}_{g}$, namely

$$
\lambda=\bigwedge^{g} \pi_{*} \Omega_{\mathrm{C}_{g} / \mathrm{M}_{g}}^{1}
$$

A Teichmüller modular form of genus $g$ and weight $h$ over $R$ is an element of

$$
\mathbf{T}_{g, h}(R)=\Gamma\left(\mathrm{M}_{g} \otimes R, \boldsymbol{\lambda}^{\otimes h}\right)
$$

Assume now that $R=k$ is a field. For a projective nonsingular variety $X$ defined over $k$, we denote by $\Omega_{k}^{1}[X]=H^{0}\left(X, \Omega_{X}^{1} \otimes k\right)$ the finite dimensional $k$-vector space of regular differential forms on $X$ defined over $k$. Let $(A, a) \in \mathrm{A}_{g} \otimes k$ be a principally polarized abelian variety of dimension $g$ defined over $k$ (resp. $C \in \mathrm{M}_{g} \otimes k$ a genus $g$ curve defined over $k$ ). We denote by

$$
\omega_{k}[A] \simeq \bigwedge^{g} \Omega_{k}^{1}[A] \quad\left(\text { resp. } \lambda_{k}[C] \simeq \bigwedge^{g} \Omega_{k}^{1}[C]\right)
$$

the $k$-vector space of sections of $\boldsymbol{\omega}$ (resp. $\boldsymbol{\lambda}$ ) over $(A, a)$ (resp. $C$ ). For $f \in \mathbf{S}_{g, h}(k)$ (resp. $f \in \mathbf{T}_{g, h}(k)$ ), and $\omega$ a basis of $\omega_{k}[A]$ (resp. $\lambda$ a basis of $\lambda_{k}[C]$ ), we put

$$
f((A, a), \omega)=f(A, a) / \omega^{\otimes h} \in k, \quad\left(\text { resp. } f(C, \lambda)=f(C) / \lambda^{\otimes h} \in k\right) .
$$


In this way a modular form defines a rule which assigns the element $f((A, a), \omega) \in k$ (resp. $f(C, \lambda))$ to every such pair $((A, a), \omega)$ (resp. $(C, \lambda))$ which depends only on $\bar{k}$-isomorphism class of the pair. With this definition, the following proposition holds, see for instance [11]:

Proposition 1.1.1. The Torelli map $t: \mathrm{M}_{g} \longrightarrow \mathrm{A}_{g}$, associating to a curve $C$ its Jacobian Jac $C$ with the canonical polarization $j$, satisfies $t^{*} \boldsymbol{\omega}=\boldsymbol{\lambda}$, and induces for any field $k$ a linear map

$$
t^{*}: \mathbf{S}_{g, h}(k)=\Gamma\left(\mathrm{A}_{g} \otimes k, \boldsymbol{\omega}^{\otimes h}\right) \longrightarrow \mathbf{T}_{g, h}(k)=\Gamma\left(\mathrm{M}_{g} \otimes k, \boldsymbol{\lambda}^{\otimes h}\right),
$$

For any curve $C / k$ of genus $g$ and any $f \in \mathbf{S}_{g, h}(k)$, one has

$$
\left[t^{*} f\right](C)=t^{*}[f(\operatorname{Jac} C, j)]
$$

i.e. for any basis $\omega$ of $\omega_{k}[\mathrm{Jac} C]$,

$$
f((\operatorname{Jac} C, j), \omega)=\left[t^{*} f\right](C, \lambda) \quad \text { if } t^{*} \omega=\lambda .
$$

Assume now that $R=k=\mathbb{C}$. Let $\mathbf{R}_{g, h}(\mathbb{C})$ be the vector-space of analytic Siegel modular forms of weight $h$ on $\mathbb{H}_{g}=\left\{\tau \in \mathbf{M}_{g}(\mathbb{C}) \mid{ }^{t} \tau=\tau, \operatorname{Im} \tau>0\right\}$, consisting of complex holomorphic functions $\phi(\tau)$ on $\mathbb{H}_{g}$ satisfying

$$
\phi(M . \tau)=\operatorname{det}(c \tau+d)^{h} \cdot \phi(\tau) \quad \text { if } M=\left(\begin{array}{ll}
a & b \\
c & d
\end{array}\right) \in \operatorname{Sp}_{2 g}(\mathbb{Z}) .
$$

To a point $\tau \in \mathbb{H}_{g}$ we associate the abelian variety $A_{\tau}=\mathbb{C}^{g} /\left(\mathbb{Z}^{g}+\tau \mathbb{Z}^{g}\right)$ with its natural principal polarization $j$. Since the tangent space to $A_{\tau}$ is canonically isomorphic to $\mathbb{C}^{g}, d z_{1} \wedge \cdots \wedge d z_{g}$ is a section of $\boldsymbol{\omega} \otimes \mathbb{C} \simeq \mathcal{O}_{\mathbb{H}_{g}} \otimes \bigwedge^{g}\left(\mathbb{C}^{g}\right)$. Thus, it induces a map from $\mathbf{R}_{g, h}(\mathbb{C})$ to $\mathbf{S}_{g, h}(\mathbb{C})$. More precisely, the following result holds [5, p. 141]:

Proposition 1.1.2. If $f \in \mathbf{S}_{g, h}(\mathbb{C})$ and $\tau \in \mathbb{H}_{g}$, let

$$
\widetilde{f}(\tau)=(2 i \pi)^{-g h} f\left(A_{\tau}, j\right) /\left(d z_{1} \wedge \cdots \wedge d z_{g}\right)^{\otimes h}
$$

where $\left(z_{1}, \ldots z_{g}\right)$ is the canonical basis of $\mathbb{C}^{g}$. The map $f \mapsto \tilde{f}$ is an isomorphism $\mathbf{S}_{g, h}(\mathbb{C}) \stackrel{\sim}{\longrightarrow} \mathbf{R}_{g, h}(\mathbb{C})$.

In the sequel we shall need some specific Siegel modular forms. We recall the definition of Thetanullwerte with characteristics

$$
\varepsilon=\left[\begin{array}{l}
\varepsilon_{1} \\
\varepsilon_{2}
\end{array}\right] \in\{0,1\}^{g} \oplus\{0,1\}^{g},
$$

given, for $\tau \in \mathbb{H}_{g}$, by

$$
\theta[\varepsilon](\tau)=\sum_{n \in \mathbb{Z}^{g}} \exp \left(i \pi\left(n+\varepsilon_{1} / 2\right) \tau^{t}\left(n+\varepsilon_{1} / 2\right)+2 i \pi\left(n+\varepsilon_{1} / 2\right)^{t}\left(\varepsilon_{2} / 2\right)\right) .
$$

Let $S_{g}$ be the set of even characteristics, that is, $\varepsilon_{1}{ }^{t} \varepsilon_{2} \equiv 0(\bmod 2)$. For $g \geq 2$ and $\tau \in \mathbb{H}_{g}$, we put $h=\left|S_{g}\right| / 2=2^{g-2}\left(2^{g}+1\right)$ and

$$
\widetilde{\chi}_{h}(\tau)=\frac{(-1)^{g h / 2}}{2^{2^{g-1}\left(2^{g}-1\right)}} \cdot \prod_{\varepsilon \in S_{g}} \theta[\varepsilon](\tau) .
$$


In [13], Igusa proves that if $g \geq 3$, then $\widetilde{\chi}_{h} \in \mathbf{R}_{g, h}(\mathbb{C})$. Starting from the analytic Siegel modular form $\tilde{\chi}_{h}$, we define, thanks to Prop. 1.1.2, a geometric Siegel modular form

$$
\chi_{h}\left(A_{\tau}\right)=(2 i \pi)^{g h} \cdot \tilde{\chi}_{h}(\tau)\left(d z_{1} \wedge \cdots \wedge d z_{g}\right)^{\otimes h} \in \mathbf{S}_{g, h}(\mathbb{C}) .
$$

Ichikawa proved several important results on this modular form that we summarize in the following proposition, see [11, Prop.3.4] and [12]:

Proposition 1.1.3. The geometric Siegel modular form $\chi_{h}$ belongs to $\mathbf{S}_{g, h}(\mathbb{Z})$. Moreover, there exists a Teichmüller modular form $\mu_{h / 2} \in \mathbf{T}_{g, h / 2}(\mathbb{Z})$ such that

$$
t^{*}\left(\chi_{h}\right)=\left(\mu_{h / 2}\right)^{2} .
$$

1.2. Action of isomorphisms. Let $k$ be any field and $\phi:\left(A^{\prime}, a^{\prime}\right) \longrightarrow(A, a)$ a $\bar{k}$-isomorphism of principally polarized abelian varieties. Choose a basis $\left(\omega_{1}, \ldots, \omega_{g}\right)$ of $\Omega_{\bar{k}}^{1}[A]$ and put $\omega=\omega_{1} \wedge \cdots \wedge \omega_{g} \in \omega_{k}[A]$. If $\gamma_{i}=\phi^{*}\left(\omega_{i}\right)$, then $\left(\gamma_{1}, \ldots, \gamma_{g}\right)$ is a basis of $\Omega^{1}{ }_{k}\left[A^{\prime}\right]$ and by invariance under $\bar{k}$-isomorphisms

$$
f((A, a), \omega)=f\left(\left(A^{\prime}, a^{\prime}\right), \gamma\right) \quad \text { where } \gamma=\gamma_{1} \wedge \cdots \wedge \gamma_{g} \in \omega_{\bar{k}}\left[A^{\prime}\right] .
$$

If $\left(\omega_{1}^{\prime}, \ldots, \omega_{g}^{\prime}\right)$ is another basis of $\Omega^{1}{ }_{\bar{k}}\left[A^{\prime}\right]$ and $\omega^{\prime}=\omega_{1}^{\prime} \wedge \cdots \wedge \omega_{g}^{\prime}$, we denote by $M_{\phi} \in \mathrm{GL}_{g}(\bar{k})$ the matrix of the basis $\left(\gamma_{i}\right)$ in the basis $\left(\omega_{i}^{\prime}\right)$. Then one proves easily:

Proposition 1.2.1. In the above notation,

$$
f((A, a), \omega)=\operatorname{det}\left(M_{\phi}\right)^{h} \cdot f\left(\left(A^{\prime}, a^{\prime}\right), \omega^{\prime}\right) .
$$

First of all, from this formula applied to the action of -1 , we deduce that, if $k$ is a field of characteristic different from 2, then $\mathbf{S}_{g, h}(k)=\{0\}$ if $g h$ is odd. From now on we assume that $g h$ is even and char $k \neq 2$.

Corollary 1.2.2. Let $(A, a)$ be a principally polarized abelian variety of dimension $g$ defined over $k$ and $f \in \mathbf{S}_{g, h}(k)$. Let $\omega_{1}, \ldots, \omega_{g}$ be a basis of $\Omega_{k}^{1}[A]$, and let $\omega=$ $\omega_{1} \wedge \cdots \wedge \omega_{g} \in \omega_{k}[A]$. Then

$$
\bar{f}(A, a)=f((A, a), \omega) \bmod ^{\times} k^{\times h} \in k / k^{\times h}
$$

does not depend on the choice of the basis of $\Omega_{k}^{1}[A]$. In particular $\bar{f}(A, a)$ is an invariant of the $k$-isomorphism class of $(A, a)$.

Corollary 1.2.3. Assume $g$ odd. Let $f \in \mathbf{S}_{g, h}(k)$ and $\phi:\left(A^{\prime}, a^{\prime}\right) \longrightarrow(A, a)$ be a non trivial quadratic twist. There exists $c \in k \backslash k^{2}$ such that $\bar{f}(A, a)=c^{h / 2} \bar{f}\left(A^{\prime}, a^{\prime}\right)$. Thus, if $\bar{f}(A, a) \neq 0$ then $\bar{f}(A, a)$ and $\bar{f}\left(A^{\prime}, a^{\prime}\right)$ do not belong to the same class in $k^{\times} / k^{\times h}$.

Proof. Assume that $\phi$ is given by a quadratic character $\varepsilon$ of $\operatorname{Gal}(\bar{k} / k)$. Then

$$
d^{\sigma}=\varepsilon(\sigma)^{g} \cdot d, \text { where } d=\operatorname{det}\left(M_{\phi}\right) \in \bar{k}, \quad \sigma \in \operatorname{Gal}(\bar{k} / k) .
$$

Since $g$ is odd, by our assumption, $h$ is even. Moreover $d^{2}=\varepsilon(\sigma) d d^{\sigma} \in k$. But $d \notin k$ since there exists $\sigma$ such that $\varepsilon(\sigma)=-1$. Using Prop. 1.2.1 we find that

$$
f((A, a), \omega)=\left(d^{2}\right)^{h / 2} f\left(\left(A^{\prime}, a^{\prime}\right), \omega^{\prime}\right) .
$$

Since $d^{2}$ is not a square in $k$, if $\bar{f}(A, a) \neq 0$ then $\bar{f}(A, a)$ and $\bar{f}\left(A^{\prime}, a^{\prime}\right)$ belong to two different classes. 
Let $(A, a)$ be a principally polarized complex abelian variety of dimension $g$ defined over $k \subset \mathbb{C}$. The period matrix of $(A, a)$ defined by a basis $\omega_{1}, \ldots, \omega_{g}$ of $\Omega_{k}^{1}[A]$ and a symplectic basis $\gamma_{1}, \ldots, \gamma_{2 g}$ of $H_{1}(A, \mathbb{Z})$ for the polarization $a$, is the Riemann matrix

$$
\Omega=\left[\begin{array}{ll}
\Omega_{1} & \Omega_{2}
\end{array}\right]=\left(\begin{array}{ccc}
\int_{\gamma_{1}} \omega_{1} & \cdots & \int_{\gamma_{2 g}} \omega_{1} \\
\vdots & & \vdots \\
\int_{\gamma_{1}} \omega_{g} & \cdots & \int_{\gamma_{2 g}} \omega_{g}
\end{array}\right) .
$$

One puts $\tau:=\Omega_{2}^{-1} \Omega_{1} \in \mathbb{H}_{3}$ in such a way that $(A, a)$ is $\mathbb{C}$-isomorphic to $A_{\tau}$. If $C$ is a complex curve of genus $g$, one uses the same notation for the period matrix of $C$ defined by a basis $\omega_{1}, \ldots, \omega_{g}$ of $\Omega_{k}^{1}[C]$, and a symplectic basis $\gamma_{1}, \ldots \gamma_{2 g}$ of $H_{1}(C, \mathbb{Z})$ for the intersection pairing. By the canonical identifications

$$
\Omega^{1}[C]=\Omega^{1}[\operatorname{Jac} C], \quad H_{1}(C, \mathbb{Z})=H_{1}(\operatorname{Jac} C, \mathbb{Z}),
$$

the period matrix of $C$ is also the period matrix of $(\operatorname{Jac} C, j)$ defined by the corresponding bases. Applying Prop. 1.2.1 with the isomorphism $z \mapsto \Omega_{2}^{-1} z$, we get the following lemma.

Proposition 1.2.4. In the above notation, let $\omega=\omega_{1} \wedge \cdots \wedge \omega_{g} \in \omega_{k}[A]$. Then

$$
f((A, a), \omega)=(2 i \pi)^{g h} \frac{\widetilde{f}(\tau)}{\operatorname{det} \Omega_{2}^{h}} .
$$

1.3. Jacobian among abelian threefolds. Serre stated in [16] and [20] the following precise form of Torelli's theorem:

Theorem 1.3.1. Let $(A, a)$ be a principally polarized abelian variety of dimension $g \geq 1$ over a field $k$, and assume that $(A, a)$ is isomorphic over $\bar{k}$ to the Jacobian of a nonsingular curve $C$. Then $C$ can be defined over $k$, and:

(i) If $C$ is hyperelliptic, there is an isomorphism, defined over $k$, from $(A, a)$ to $(\mathrm{Jac} C, j)$.

(ii) If $C$ is not hyperelliptic, there exists a quadratic character

$$
\varepsilon: \operatorname{Gal}\left(k^{\text {sep }} / k\right) \longrightarrow\{ \pm 1\}
$$

and an isomorphism, defined over $k$, from the twisted abelian variety $(A, a)_{\varepsilon}$ to $(\mathrm{Jac} C, j)$. Hence, $(A, a)$ is $k$-isomorphic to a Jacobian if and only if $\varepsilon$ is trivial.

We restrict to the case where $k \subset \mathbb{C}$ and we now give a formula for $\varepsilon$. In order to do so, we need to recall some geometric results by Igusa. Denote by $\widetilde{\Sigma}_{140}$ the modular form defined by the thirty-fifth elementary symmetric function of the eighth power of the even Thetanullwerte. Recall that a principally polarized abelian variety $(A, a)$ is decomposable if it is a product of principally polarized abelian varieties of lower dimension, and indecomposable otherwise. In his beautiful paper [13], Igusa proves the following result [loc. cit., Lem. 10 and 11].

Theorem 1.3.2. If $\tau \in \mathbb{H}_{3}$, then:

(i) $\left(A_{\tau}, j\right)$ is decomposable if $\widetilde{\chi}_{18}(\tau)=\widetilde{\Sigma}_{140}(\tau)=0$.

(ii) $\left(A_{\tau}, j\right)$ is a hyperelliptic Jacobian if $\widetilde{\chi}_{18}(\tau)=0$ and $\widetilde{\Sigma}_{140}(\tau) \neq 0$.

(iii) $\left(A_{\tau}, j\right)$ is a non hyperelliptic Jacobian if $\widetilde{\chi}_{18}(\tau) \neq 0$. 
We are now able to prove our main result which can be seen as an arithmetic analogue of Igusa's result.

Theorem 1.3.3. Let $(A, a)$ be a principally polarized abelian threefold defined over $k \subset \mathbb{C}$. Let $\left(\omega_{1}, \omega_{2}, \omega_{3}\right)$ be any basis of $\Omega_{k}^{1}[A]$ and $\left(\gamma_{1}, \ldots, \gamma_{6}\right)$ a symplectic basis of $H_{1}(A, \mathbb{Z})$ for the polarization a. Let $\Omega=\left[\Omega_{1} \Omega_{2}\right]$ be the period matrix defined by these bases, and $\tau=\Omega_{2}^{-1} \Omega_{1}$.

(i) If $\widetilde{\Sigma}_{140}(\tau)=0$ and $\widetilde{\chi}_{18}(\tau)=0$ then $(A, a)$ is decomposable over $\bar{k}$. In particular it is not a Jacobian.

(ii) If $\widetilde{\Sigma}_{140}(\tau) \neq 0$ and $\widetilde{\chi}_{18}(\tau)=0$ then there exists a hyperelliptic curve $C / k$ such that $(\mathrm{Jac} C, j) \simeq(A, a)$.

(iii) If $\widetilde{\chi}_{18}(\tau) \neq 0$ then $(A, a)$ is isomorphic to a non hyperelliptic Jacobian if and only if

$$
\chi_{18}:=\chi_{18}((A, a), \omega)=(2 i \pi)^{54} \frac{\widetilde{\chi}_{18}(\tau)}{\operatorname{det} \Omega_{2}^{18}}
$$

is a square in $k$, with $\omega=\omega_{1} \wedge \omega_{2} \wedge \omega_{3} \in \omega_{k}[A]$.

Proof. Only the third point is new. Indeed, the first and second points directly follow from Th. 1.3.2 and Th. 1.3.1. Suppose now that $(A, a)$ is isomorphic over $k$ to the Jacobian of a non hyperelliptic genus 3 curve $C / k$. Using successively Prop. 1.1.1 and Prop. 1.1.3, we get

$$
\chi_{18}((A, a), \omega)=t^{*}\left(\chi_{18}\right)(C, \lambda)=\mu_{9}(C, \lambda)^{2} \in k^{\times 2},
$$

with $\lambda=t^{*} \omega$. Hence, the desired expression is a square in $k^{\times}$. Its analytic expression on the right hand side of (iii) is a direct application of Prop. 1.2.4.

On the contrary, Cor. 1.2.3 shows that if $(A, a)$ is a quadratic twist of a Jacobian $\left(A^{\prime}, a^{\prime}\right)$ then there exists a non square $c \in k$ such that

$$
\bar{\chi}_{18}(A, a)=c^{9} \cdot \bar{\chi}_{18}\left(A^{\prime}, a^{\prime}\right) .
$$

As we have just proved that $\bar{\chi}_{18}\left(A^{\prime}, a^{\prime}\right)$ is a square in $k^{\times} / k^{\times 18}$, this implies that $\chi_{18}((A, a), \omega)$ is not.

Corollary 1.3.4. In the notation of Th. 1.3.3, the quadratic character $\varepsilon$ of $\operatorname{Gal}(\bar{k} / k)$ introduced in Th. 1.3 .1 is given by $\varepsilon(\sigma)=d^{\sigma} / d$, with $d=\sqrt{\chi_{18}}$, and with an arbitrary choice of the square root.

\section{Invariants and Klein's formula}

Let $d>0$ be an integer. In this section $k$ is an algebraically closed field of characteristic coprime with $d$.

2.1. Geometric invariants for nonsingular plane curves. We first review some classical invariant theory. Let $E$ be a vector space of dimension $n$ over $k$. The left regular representation $r$ of $\mathrm{GL}(E)$ on the vector space $\mathrm{X}_{d}=\operatorname{Sym}^{d}\left(E^{*}\right)$ of forms of degree $d$ on $E$ is given by

$$
r(u): F(x) \mapsto(u \cdot F)(x)=F(u x)
$$


for $u \in \mathrm{GL}(E), F \in \mathrm{X}_{d}$ and $x \in E$. If $U$ is an open subset of $\mathrm{X}_{d}$ stable under $r$, we still denote by $r$ the left regular representation of GL $(E)$ on the $k$-algebra $\mathcal{O}(U)$ of regular functions on $U$, in such a way that

$$
r(u): \Phi(F) \mapsto(u \cdot \Phi)(F)=\Phi(u \cdot F),
$$

if $u \in \mathrm{GL}(E), \Phi \in \mathcal{O}(U)$ and $F \in U$. If $h \in \mathbb{Z}$, we denote by $\mathcal{O}_{h}(U)$ the subspace, stable under $r$, of homogeneous elements of degree $h$. An element $\Phi \in \mathcal{O}_{h}(U)$ is an invariant of degree $h$ on $U$ if $u \cdot \Phi=\Phi$ for every $u \in \mathrm{SL}(E)$, and we denote by $\operatorname{lnv}_{h}(U)$ the subspace of invariants of degree $h$ on $U$. Hence, if $\Phi \in \mathcal{O}(U)$, and if $w$ and $n$ are two integers such that $h d=n w$, then $\Phi \in \operatorname{lnv}_{h}(U)$ if and only if

$$
u \cdot \Phi=(\operatorname{det} u)^{w} \Phi \text { for every } u \in \operatorname{GL}(E),
$$

and we call $w$ the weight of $\Phi$. Let $F \in \mathrm{X}_{d}$, and denote by $q_{1}, \ldots, q_{n}$ the partial derivatives of $F$. The discriminant of $F$ is

$$
\operatorname{Disc} F=c_{n, d}^{-1} \operatorname{Res}\left(q_{1}, \ldots, q_{n}\right), \quad \text { with } \quad c_{n, d}=d^{\left((d-1)^{n}-(-1)^{n}\right) / d},
$$

where $\operatorname{Res}\left(q_{1}, \ldots, q_{n}\right)$ is the multivariate resultant of the forms $q_{1}, \ldots q_{n}[6$, p. 426], the coefficient $c_{n, d}$ being chosen according to [20]. We refer to [15] for a detailed study of the discriminant of a ternary form, and the computation of the discriminant of a Ciani quartic.

From now on we assume $\operatorname{dim} E=n=3$. The universal curve over $\mathrm{X}_{d}$ is the variety

$$
\mathrm{Y}_{d}=\left\{(F, x) \in \mathrm{X}_{d} \times \mathbb{P}^{2} \mid F(x)=0\right\} .
$$

The nonsingular locus of $\mathrm{X}_{d}$ is the principal open set

$$
\mathrm{X}_{d}^{0}=\left(\mathrm{X}_{d}\right)_{\text {Disc }}=\left\{F \in \mathrm{X}_{d} \mid \operatorname{Disc}(F) \neq 0\right\} .
$$

If $\mathrm{Y}_{d}^{0}$ is the universal curve over the nonsingular locus $\mathrm{X}_{d}^{0}$, the projection is a smooth surjective $k$-morphism

$$
\pi: \mathrm{Y}_{d}^{0} \longrightarrow \mathrm{X}_{d}^{0}
$$

whose fibre over $F$ is the non singular plane curve $C_{F}$. If $F \in \mathrm{X}_{d}^{0}(k)$, we recall the usual way to write down explicitly the classical basis of $\Omega_{k}^{1}\left[C_{F}\right]=H^{0}\left(C_{F}, \Omega_{C_{F}}^{1} \otimes k\right)$, see $[2$, p. 630]. Let

$$
\eta^{(1)}=\frac{f\left(x_{2} d x_{3}-x_{3} d x_{2}\right)}{q_{1}}, \quad \eta^{(2)}=\frac{f\left(x_{3} d x_{1}-x_{1} d x_{3}\right)}{q_{2}}, \quad \eta^{(3)}=\frac{f\left(x_{1} d x_{2}-x_{2} d x_{1}\right)}{q_{3}},
$$

where $q_{1}, q_{2}, q_{3}$ are the partial derivatives of $F$, and where $f \in \mathrm{X}_{d-3}$. The forms $\eta^{(i)}$ glue together and define a regular differential form $\eta_{f}(F) \in \Omega_{k}^{1}\left[C_{F}\right]$. Since $\operatorname{dim} \mathrm{X}_{d-3}=$ $(d-1)(d-2) / 2=g$, the linear map $f \mapsto \eta_{f}(F)$ defines an isomorphism

$$
\mathrm{X}_{d-3} \stackrel{\sim}{\longrightarrow} \Omega_{k}^{1}\left[C_{F}\right] .
$$

We denote $\eta_{1}, \ldots, \eta_{g}$ the sequence of sections obtained by substituting for $f$ in $\eta_{f}$ the $g$ members of the canonical basis of $\mathbf{X}_{d-3}$, enumerated according to the lexicographic order. Then $\eta=\eta_{1} \wedge \cdots \wedge \eta_{g}$ is a section of

$$
\boldsymbol{\alpha}=\bigwedge^{g} \pi_{*} \Omega_{\mathrm{Y}_{d}^{0} / \mathbf{X}_{d}^{0}}^{1},
$$

the Hodge bundle on $\mathrm{X}_{d}^{0}$. The map $u: x \mapsto u x$ induces an isomorphism

$$
u: C_{u \cdot F} \stackrel{\sim}{\longrightarrow} C_{F}
$$


Hence, it has a natural action $u^{*}: \Omega_{k}^{1}\left[C_{F}\right] \rightarrow \Omega_{k}^{1}\left[C_{u \cdot F}\right]$ on the differentials and therefore, on the sections of $\boldsymbol{\alpha}^{h}$, for $h \in \mathbb{Z}$. More specifically, if $s \in \Gamma\left(\mathbf{X}_{d}^{0}, \boldsymbol{\alpha}^{\otimes h}\right)$, one can write $s=\Phi \cdot \eta^{\otimes h}$ with $\Phi \in \mathcal{O}\left(\mathrm{X}_{d}^{0}\right)$; for $F \in \mathrm{X}_{d}^{0}$, one has

$$
u^{*} s(F)=\Phi(F) \cdot\left(u^{*} \eta(F)\right)^{\otimes h} .
$$

The proof of the following lemma is left to the reader.

Lemma 2.1.1. For any $u \in G$ and any $F \in \mathbf{X}_{d}^{0}$, the section $\eta \in \Gamma\left(\mathbf{X}_{d}^{0}, \boldsymbol{\alpha}\right)$ satisfies,

$$
u^{*} \eta(F)=\operatorname{det}(u)^{w_{0}} \cdot \eta(u \cdot F), \quad \text { with } w_{0}=\left(\begin{array}{l}
d \\
3
\end{array}\right)=\frac{d g}{3} \in \mathbb{N} .
$$

For any $h \in \mathbb{Z}$, we denote by $\Gamma\left(\mathrm{X}_{d}^{0}, \boldsymbol{\alpha}^{\otimes h}\right)^{G}$ the subspace of sections $s \in \Gamma\left(\mathrm{X}_{d}^{0}, \boldsymbol{\alpha}^{\otimes h}\right)$ such that $u^{*} s(F)=s(u \cdot F)$ for every $u \in G$ and $F \in \mathrm{X}_{d}^{0}$.

Proposition 2.1.2. Let $h \geq 0$ be an integer. The linear map

$$
\Phi \mapsto \rho(\Phi)=\Phi \cdot \eta^{\otimes h}
$$

is an isomorphism

$$
\rho: \operatorname{lnv}_{g h}\left(\mathbf{X}_{d}^{0}\right) \stackrel{\sim}{\longrightarrow} \Gamma\left(\mathbf{X}_{d}^{0}, \boldsymbol{\alpha}^{\otimes h}\right)^{G} .
$$

Proof. Let $\Phi \in \operatorname{lnv}_{g h}\left(\mathrm{X}_{d}^{0}\right), s=\rho(\Phi)=\Phi \cdot \eta^{\otimes h}$, and $w=d g h / 3$, the weight of $\Phi$. Then using Lem. 2.1.1,

$$
\begin{aligned}
u^{*} s(F)=\Phi(F) \cdot & \left(u^{*} \eta(F)\right)^{\otimes h}=\Phi(F) \cdot \operatorname{det}(u)^{w_{0} h} \cdot \eta(u \cdot F)^{\otimes h} \\
& =\operatorname{det}(u)^{w} \Phi(F) \cdot \eta(u \cdot F)^{\otimes h}=\Phi(u \cdot F) \cdot \eta(u \cdot F)^{\otimes h}=s(u \cdot F) .
\end{aligned}
$$

Hence, $\rho(\Phi) \in \Gamma\left(\mathbf{X}_{d}^{0}, \boldsymbol{\lambda}^{\otimes h}\right)^{G}$. Conversely, the inverse of $\rho$ is the map $s \mapsto s / \eta^{\otimes h}$, and this proves the proposition.

2.2. Modular forms as invariants. Let $d>2$ be an integer and $g=\left(\begin{array}{c}d-1 \\ 2\end{array}\right)$. Since the fibres of $\mathrm{Y}_{d}^{0} \longrightarrow \mathrm{X}_{d}^{0}$ are nonsingular non hyperelliptic plane curves of genus $g$, by the universal property of $\mathrm{M}_{g}$ we get a morphism

$$
p: \mathrm{X}_{g}^{0} \longrightarrow \mathrm{M}_{g} \text {. }
$$

and $p^{*} \boldsymbol{\lambda}=\boldsymbol{\alpha}$ by construction. This induces a linear map

$$
p^{*}: \mathbf{T}_{g, h}(k) \longrightarrow \Gamma\left(\mathbf{X}_{d}^{0}, \boldsymbol{\alpha}^{\otimes h}\right) .
$$

Moreover, for $u \in G$, since $u: C_{u \cdot F} \rightarrow C_{F}$ is an isomorphism, we get the following commutative diagram

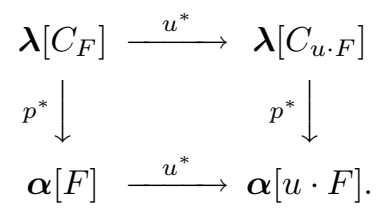

For any $f \in \mathbf{T}_{g, h}(k)$, the modular invariance of $f$ means that

$$
u^{*} f\left(C_{F}\right)=f\left(C_{u \cdot F}\right)
$$

Then

$$
u^{*}\left[\left(p^{*} f\right)(F)\right]=u^{*}\left[p^{*}\left(f\left(C_{F}\right)\right)\right]=p^{*}\left[u^{*} f\left(C_{F}\right)\right]=p^{*}\left[f\left(C_{u \cdot F}\right)\right]=\left(p^{*} f\right)(u \cdot F),
$$


and this means that $p^{*} f \in \Gamma\left(\mathbf{X}_{d}^{0}, \boldsymbol{\alpha}^{\otimes h}\right)^{G}$. If $g=3$ then $p^{*}$ is a linear isomorphism. Combining this result with Prop. 2.1.2, we obtain:

Proposition 2.2.1. For any integer $h \geq 0$, the linear map $\sigma=\rho^{-1} \circ p^{*}$ is a homomorphism:

such that

$$
\mathbf{T}_{g, h}(k) \longrightarrow \operatorname{lnv}_{g h}\left(\mathrm{X}_{d}^{0}\right)
$$

$$
\sigma(f)(F)=f\left(C_{F}, \lambda\right)
$$

with $\lambda=\left(p^{*}\right)^{-1} \eta$, for any $F \in \mathbf{X}_{d}^{0}$ and any section $f \in \mathbf{T}_{g, h}(k)$. If $g=3$, then $\sigma$ is an isomorphism.

We finally make a link between invariants and analytic Siegel modular forms. Let $F \in X_{d}^{0}(\mathbb{C})$ and $\left(\eta_{1}, \ldots, \eta_{g}\right)$ the basis of regular differentials on $C_{F}$ defined in $\S 2.1$. Let $\left(\gamma_{1}, \ldots \gamma_{2 g}\right)$ be a symplectic basis of $H_{1}(C, \mathbb{Z})$ for the intersection pairing. Let $\Omega=\left[\Omega_{1} \Omega_{2}\right]$ the period matrix of $C_{F}$ defined by these bases, and $\tau=\Omega_{2}^{-1} \Omega_{1}$.

Corollary 2.2.2. Let $f \in \mathbf{S}_{g, h}(\mathbb{C})$ be a geometric Siegel modular form, $\tilde{f} \in \mathbf{R}_{g, h}(\mathbb{C})$ the corresponding analytic modular form, and $\Phi=\sigma\left(t^{*} f\right)$ the corresponding invariant. In the above notation,

$$
\Phi(F)=(2 i \pi)^{g h} \frac{\widetilde{f}(\tau)}{\operatorname{det} \Omega_{2}^{h}} .
$$

Proof. Let $\lambda=\left(p^{*}\right)^{-1}(\eta)$ and $\omega=\left(t^{*}\right)^{-1}(\lambda)$. From Prop. 1.1.1 and 2.2.1, we deduce

$$
\Phi(F)=\left(t^{*} f\right)\left(C_{F}, \lambda\right)=f\left(\operatorname{Jac} C_{F}, \omega\right),
$$

and Prop. 1.2.4 give the result, since $\Omega$ is also the period matrix of $\operatorname{Jac} C_{F}$.

We are now ready to give a proof of the following result [14, Eq. 118, p. 462]:

Theorem 2.2.3 (Klein's formula). Let $F \in \mathrm{X}_{4}^{0}(\mathbb{C})$ and $C_{F}$ be the corresponding smooth plane quartic. Let $\left(\eta_{1}, \eta_{2}, \eta_{3}\right)$ be the classical basis of $\Omega_{\mathbb{C}}^{1}\left[C_{F}\right]$ and $\left(\gamma_{1}, \ldots \gamma_{6}\right)$ be a symplectic basis of $H_{1}\left(C_{F}, \mathbb{Z}\right)$ for the intersection pairing. Let $\Omega=\left[\Omega_{1} \Omega_{2}\right]$ the period matrix of $C_{F}$ defined by these bases, and $\tau=\Omega_{2}^{-1} \Omega_{1}$. Then

$$
\operatorname{Disc}(F)^{2}=(2 \pi)^{54} \frac{\tilde{\chi}_{18}(\tau)}{\operatorname{det}\left(\Omega_{2}\right)^{18}} .
$$

Proof. Cor. 2.2.2 shows that for any $F \in \mathrm{X}_{4}^{0}$ the invariant $I=\sigma \circ t^{*}\left(\chi_{18}\right)$ satisfies

$$
I(F)=(2 i \pi)^{54} \frac{\tilde{\chi}_{18}(\tau)}{\operatorname{det} \Omega_{2}^{18}} .
$$

Moreover Th. 1.3.2(iii) shows that $I(F) \neq 0$ for all $F \in \mathrm{X}_{4}^{0}$. Since the discriminant is an irreducible polynomial, as immediate consequence of Hilbert's Nullstellensatz we get that $I=c \operatorname{Disc}^{n}$ with $c \in \mathbb{C}^{\times}$a constant and $n \in \mathbb{N}$. Since $I$ is an invariant of weight 54 and Disc an invariant of weight $27, n=2$. Finally, it is proven in [15, Cor. 4.2 ] that Klein's formula holds true for any Ciani quartic with $c=1$.

Remark 2.2.4. Th. 2.2.3 implies that

$$
\mu_{9}\left(C_{F}, \lambda\right)= \pm \operatorname{Disc} F .
$$

This might be deduced from the definition of $\mu_{9}$, although it seems that this fact was not observed before. 
2.3. Beyond genus 3. First of all, note that an analogue of Klein's formula has been derived in the hyperelliptic case by Lockhart [17] and also by Guàrdia [8]. Their formula is a direct consequence of Thomae's formula [21]. Now, it is natural to try to extend the preceding results to the case $g>3$. For Klein's formula and $g=4$, Klein himself, in the footnote of p. 462 in [14], gives the amazing formula

$$
\frac{\tilde{\chi}_{68}(\tau)}{\operatorname{det}\left(\Omega_{2}\right)^{68}}=c \cdot \Delta(C)^{2} \cdot T(C)^{8} .
$$

Here $\tau=\Omega_{2}^{-1} \Omega_{1}$, with $\Omega=\left[\Omega_{1} \Omega_{2}\right]$ a period matrix of a genus 4 non hyperelliptic curve $C$ given in $\mathbb{P}^{3}$ as an intersection of a quadric $Q$ and a cubic surface $E$. The elements $\Delta(C)$ and $T(C)$ are defined in classical invariant theory as, respectively, the discriminant of $Q$ and the tact invariant of $Q$ and $E$ (see [19, p.122]). No such formula seems to be known in the non hyperelliptic case for $g>4$.

Let us now look at what happens when we try to apply Serre's approach for $g>3$. To begin with, when $g$ is even, we cannot use Cor. 1.2.2 to distinguish between quadratic twists. Let us assume that $g$ is odd. Cor. 1.2.3 shows that there exists $c \in k \backslash k^{2}$ such that

$$
\bar{\chi}_{h}\left(A^{\prime}, a^{\prime}\right)=c^{h / 2} \cdot \bar{\chi}_{h}(A, a)
$$

for a Jacobian $(A, a)$ and a quadratic twist $\left(A^{\prime}, a^{\prime}\right)$. What enabled us to distinguish between the two when $g=3$ is that $h / 2=9$ is odd. However as soon as $g>3$, the 2 -valuation of $h / 2$ is $g-3>0$, so it is not enough for $\bar{\chi}_{h}(A)$ to be a square in $k$ to make a distinction between $A$ and $A^{\prime}$. It must rather be a $2^{g-2}$-th power in $k$. It can be easily seen from the proof of $\left[22\right.$, Th.1] that $t^{*}\left(\chi_{h}\right)$ does not admit a fourth root. According to [1] or [23] this implies $\bar{\chi}_{h}(A, a)$ is not a $2^{g-2}$-th power in $k$ for infinitely many Jacobians $(A, a)$ defined over number fields $k$. So we can no longer use the modular form $\chi_{h}$ to characterize Jacobians over $k$.

\section{References}

[1] Bilu, Y. F. Letter to the authors, dated February 16, 2008.

[2] Brieskorn, E.; Knörrer, H. Plane Algebraic Curves. Birkhäuser Verlag, 1986.

[3] Chai, C. L. Siegel moduli schemes and their compactifications over $\mathbb{C}$. Arithmetic geometry (Storrs, Conn., 1984), 231-251, Springer, New York, 1986.

[4] Deligne, P.; Mumford, D. The irreducibility of the space of curves of given genus. Inst. Hautes tudes Sci. Publ. Math. 36 (1969), 75-109.

[5] Faltings, G.; Chai, C.-L. Degeneration of abelian varieties. Ergebnisse der Mathematik und ihrer Grenzgebiete (3), 22. Springer, Berlin, 1990.

[6] Gel'fand, I.M.; Kapranov, M.M.; Zelevinsky, A.V. Discriminants, resultants, and multidimensional determinants. Birkhäuser, Boston, (1994).

[7] van der Geer, G. Siegel modular forms and their applications. The 1-2-3 of modular forms, 181-245, Universitext, Springer, Berlin, 2008.

[8] Guàrdia, G. Jacobian nullwerte and algebraic equations. J. Algebra 253 (2002), 112-132.

[9] Hoyt, W.L. On products and algebraic families of Jacobian varieties. Ann. of Math. 77, (1963), 415-423.

[10] Ichikawa, T. Teichmüller modular forms of degree 3. Amer. J. Math. 117 (1995), no. 4, 10571061.

[11] Ichikawa, T. Theta constants and Teichmüller modular forms. J. Number Theory 61 (1996), no. $2,409-419$.

[12] Ichikawa, T. Generalized Tate curve and integral Teichmüller modular forms. Amer. J. Math. 122 (2000), no. 6, 1139-1174.

[13] Igusa, J.-I. Modular forms and projective invariants. Amer. J. Math, 89, (1967), 817-855. 
[14] Klein, F. Zur Theorie der Abelschen Funktionen. Math. Annalen, 36 (1889-90), 1-83; = Gesammelte mathematische Abhandlungen XCVII, 388-474.

[15] Lachaud, G.; Ritzenthaler, C. On a conjecture of Serre on abelian threefolds. Algebraic Geometry and its applications (Papeete, 2007), 88-115. Series on Number Theory and Its Applications 5. World Scientific, Hackensack, NJ, 2008.

[16] Lauter, K. Geometric methods for improving the upper bounds on the number of rational points on algebraic curves over finite fields, with an appendix by J. P. Serre. Journal of Algebraic Geometry 10 (2001), 19-36.

[17] Lockhart, P. On the discriminant of a hyperelliptic curve. Trans. Amer. Math. Soc. 342, (1994), $729-752$.

[18] Oort, F.; Ueno, K. Principally polarized abelian varieties of dimension two or three are Jacobian varieties. J. Fac. Sci. Univ. Tokyo Sect. IA Math., 20, (1973), 377-381.

[19] Salmon, G. Traité de géométrie analytique à trois dimensions. Troisième partie. Ouvrage traduit de l'anglais sur la quatrième édition, Paris, 1892.

[20] Serre, J.-P. Two letters to Jaap Top. Algebraic Geometry and its applications (Tahiti, 2007) 84-87. Series on Number Theory and Its Applications 5. World Scientific, Hackensack, NJ, 2008.

[21] Thomae, J. Beitrag zur Bestimmung von $\theta(0,0, \ldots, 0)$ durch die Klassenmoduln algebraischer Funktionen, J. Reine Angew. Math. 71, (1870), 201-222.

[22] Tsuyumine, S. Thetanullwerte on a moduli space of curves and hyperelliptic loci. Math. Z. 207 (1991), 539-568.

[23] Xarles, X. Letter to the authors, dated February 18, 2008.

Gilles Lachaud

Institut DE MathÉmatiques DE LUMiny

Université AiX-Marseille - CNRS

Luminy Case 907, 13288 Marseille Cedex 9 - FRANCE

E-mail address: lachaud@iml.univ-mrs.fr

Christophe Ritzenthaler

Institut De Mathématiques De Luminy

E-mail address: ritzent@iml.univ-mrs.fr

AleXey ZyKin

Institut De Mathématiques DE Luminy AND

Mathematical Institute of the Russian Academy of Sciences

Laboratoire Poncelet (UMI 2615)

E-mail address: zykin@iml.univ-mrs.fr 